\title{
Importance of Vector Velocity Echocardiography and NT-proBNP in Identifying Individuals with Preclinical Hypertrophic Cardiomyopathy
}

\author{
Li Yu¹, Qichang Zhou ${ }^{1, *}$, Xiangdang Long ${ }^{2}$, Qinghai Peng ${ }^{1}$, and Zurong Yang ${ }^{1}$ \\ ${ }^{1}$ Department of Ultrasound Diagnosis, Second Xiangya Hospital of Central South University, Changsha, China \\ ${ }^{2}$ Department of Ultrasound Diagnosis, People's Hospital of Hunan Province, Changsha, China
}

*Corresponding author: Qichang Zhou, MD, PhD, Department of Ultrasound Diagnosis, Second Xiangya Hospital of Central South University, Changsha, 410000, China, Tel: 86-0731-85292140; E-mail: zhouqichang@csu.edu.cn

Received: 07 Sep, 2021 | Accepted: 11 Oct, 2021 | Published: 20 Oct, 2021

Citation: Yu L, Zhou Q, Long X, Peng Q, Yang Z (2021) Importance of Vector Velocity Echocardiography and NT-proBNP in Identifying Individuals with Preclinical Hypertrophic Cardiomyopathy. J Heart Health 7(2): dx.doi.org/10.16966/2379-769X.163

Copyright: (c) 2021 Yu L, et al. This is an open-access article distributed under the terms of the Creative Commons Attribution License, which permits unrestricted use, distribution, and reproduction in any medium, provided the original author and source are credited.

\begin{abstract}
Background and aim: Familial HCM gene mutation carriers without overt left ventricular hypertrophy (gene positive/phenotype negative G+/P-) can harbor subclinical changes in cardiovascular structure and function that precede the development of Hypertrophic Cardiomyopathy (HCM). Conventional echocardiography parameter ejection fraction is normal in $\mathrm{HCM}$ patients. We sought to investigate whether Velocity Vector Imaging (VVI) and NT-proBNP are more sensitive to evaluate heart function.
\end{abstract}

Methods: We studied eighteen HCM families with pathogenic mutations, 45 patients with overt $\mathrm{HCM}$ (gene positive/phenotype positive $(\mathrm{G}+/ \mathrm{P}+)$ ), 40 patients without myocardial hypertrophy (gene positive/phenotype negative $\mathrm{G}+/ \mathrm{P}-$ )), and 48 healthy controls. Conventional echocardiography and Velocity Vector Imaging (VVI) were

performed, and blood levels of N-Terminal pro-Brain Natriuretic Peptide (NT-proBNP) were analyzed.

Results: Although the longitudinal strain of the basal inferoseptum and basal anteroseptum was lower in G+/P-patients than in controls, while the basal and middle inferolateral longitudinal strains were significantly higher. Compared with the controls, $\mathrm{G}+\mathrm{P}+$ patients had significantly lower global and segmental longitudinal and radial strains. The NT-proBNP levels were higher in G+/P+ patients than in G+/P-people and controls.

Conclusions: Sarcomere gene mutation carriers without overt left ventricular hypertrophy have subclinical segmental systolic dysfunction. Velocity vector imaging is feasible for differentiating $\mathrm{HCM}$ and $\mathrm{G}+/ \mathrm{P}$ - patients from controls.

Keywords: NT-proBNP; Preclinical hypertrophic cardiomyopathy; Strain; Velocity vector imaging

\section{Introduction}

Hypertrophic Cardiomyopathy (HCM) is one of the most common autosomal dominant cardiovascular diseases and is the primary cause of sudden death in young people and athletes. In most patients, gene mutation is the primary cause of HCM. Most mutations are sarcomere protein gene mutations encoding the myocardium that exhibit autosomal dominant inheritance [1]. HCM demonstrates obvious family clustering, and the genetic probability is $50 \%$. According to statistics, the proportion of patients with familial HCM who eventually develop HCM is $40 \%-100 \%$ [2]. Early recognition of and intervention for cardiac function changes are particularly important.

Familial HCM gene mutation carriers without overt left ventricular hypertrophy (gene positive/phenotype negative G+/P-) may experience syncope and have other abnormal performance, including abnormal electrocardiogram (ECG) repolarization, and they may develop subclinical changes in cardiac function before developing myocardial hypertrophy. Therefore, it is urgent to identify these patients by imaging methods.

Velocity Vector Imaging (VVI) is based on two-dimensional grayscale images that track the spatial motion of cardiovascular tissue to show echo spots. The tracking of multiple regional myocardial segments is performed simultaneously. The velocity and displacement of the regional myocardium are displayed quantitatively as a curve. VVI can be used to analyze the movement and deformation of the myocardium, and it is possible to detect fine space and time distinctions in cardiac deformation in different myocardial segments during systole and diastole [3-7]. Therefore, VVI is valuable for evaluating regional and global cardiac function.

Many studies have shown that VVI is potentially viable for assessing myocardial function [6]. The N-terminal pro-brain natriuretic peptide (NT-proBNP) level may be related to cardiovascular damage, reflecting ventricular function [8]. However, there are no VVI 
parameters for the Left Ventricle (LV), globally or segmentally, in preclinical HCM. We aimed to evaluate changes in the long and shortaxis function of the LV using VVI combined with NT-proBNP levels.

\section{Methods}

\section{Study population}

A total of 96 unrelated HCM patients who were diagnosed in our hospital from March 2016 to April 2019 were selected for gene detection, and $45 \mathrm{HCM}$ patients who were carrying sarcomere gene mutations were selected as the gene positive/phenotype positive $(\mathrm{G}+/ \mathrm{P}+)$ group. Gene detection and conventional echocardiography were performed on the first-degree relatives of 45 unrelated patients (i.e., parents, children, siblings of the same parent). According to the examination results, 40 patients with HCM sarcomere mutation genes but no ventricular wall hypertrophy were selected as the gene positive phenotype negative (G+/P-) group. At the same time, 48 healthy ageand gender-matched subjects without cardiological disorders were selected as normal controls.

The diagnostic criterion of HCM is that the thickness of the left ventricular wall in one or more myocardial segments is greater than or equal to $15 \mathrm{~mm}$. It was necessary to exclude myocardial hypertrophy due to athletics, metabolic diseases, congenital heart diseases and other systemic diseases. In patients with a clear family history, an unexplained left ventricular wall thickness of one or more myocardial segments $\geq 13 \mathrm{~mm}$ was observed [1]

All G+/P+ individuals had interventricular septum thickening, with or without other left ventricular wall thickening. Before examination, $\beta$-blockers, calcium antagonists and angiotensin-converting enzyme inhibitors were stopped for at least 24 hours. The exclusion criteria were as follows: 1) patients with ventricular wall hypertrophy caused by hypertension, coronary heart disease, diabetes mellitus, valvular disease, congenital heart disease, pulmonary heart disease, metabolic disease or other factors, as well as athletes with cardiac hypertrophy, were excluded after obtaining a medical history and performing a physical examination, ECG and echocardiography; 2) patients with HCM whose left ventricular ejection fraction was less than $50 \%$; 3) accepted patients with HCM who underwent percutaneous septal myocardial ablation, surgical septal myectomy or permanent pacemaker implantation or experienced atrial fibrillation.

The inclusion criteria for the G+/P- group were as follows: 1) carrier of a sarcomere mutation gene verified by gene generation; 2) maximum Left Ventricular Wall Thickness (LVMWT) less than $13 \mathrm{~mm}$ detected by echocardiography. The exclusion criteria were as follows: 1) diabetes mellitus and hypertension; 2) cardiac muscle noncompaction and amyloidosis; 3) metabolic diseases and other systemic diseases; 4) significant pulmonary lesions; 5) treadmill test, coronary angiography or coronary artery Computed Tomography (CT) results indicating coronary heart disease, which was definitively diagnosed by imaging; 6) congenital heart disease; and 7) moderate and severe valve stenosis and regurgitation detected by echocardiography.

This cross-sectional study was conducted with the permission of the Institutional Ethics Committee. All subjects provided written informed consent.

\section{Conventional echocardiography}

For the echocardiography recordings, all subjects laid on their left side. Three short-axis views (mitral valve level, papillary muscle level and apical level) and three long-axis views (apical three-chamber view, apical two-chamber view and apical four-chamber view) of the LV were obtained on a Siemens S2000 ultrasound system (Axius, Siemens Medical Solutions, Malvern, PA, USA) with a 4Px probe (2.75-4.25 $\mathrm{MHz}$ ). All images and clips were stored on an echocardiographic machine for analysis.

The Interventricular Septal Thickness in Diastole (IVSD) and Left Atrial Diameter (LAD) were detected in the parasternal long-axis view. The LVMWT was measured in diastole in the basal, mid and apical short-axis views and in the apical long-axis view.

The Left Atrial Volume (LAV), Left Ventricular End-Diastolic Volume (LVEDV) and Left Ventricular End-Systolic Volume (LVESV) were measured by the Simpson biplane method in the apical twochamber and four-chamber views. The Left Atrial Volume Index (LAVI) was calculated as LAVI $=$ LAV/Body Surface Area (BSA).

The Left Ventricular Outflow Tract Pressure Gradient (LVOT-PG) was measured by Continuous-Wave Doppler (CW), and the sampling line was placed at the stenosis of the left ventricular outflow tract. In the apical four-chamber view, E/A was measured by Pulsed-wave Doppler (PW). The Ejection Fraction (EF) was estimated using the Simpson biplane method.

All recordings were performed by professional sonographers. All conventional echocardiography parameters were read offline. All recordings were performed by professional sonographers in accordance with the operational guidelines of the American Society of Echocardiography [9].

\section{Velocity vector imaging echocardiography}

Movie clips were recorded in 3 cardiac cycles and stored, and three apical views of the LV were analyzed offline using VVI software (Axius, Siemens Medical Solutions). A line was fitted along the internal surface of the LV endocardium at end-diastole. We used a frame-by-frame image tracking mode to estimate the movement of the myocardium. The acoustic marker of the myocardium was accurately identified and automatically tracked during several consecutive frames.

The longitudinal strain, circumferential strain, and radial strain curves were measured for each LV segment using long-axis and shortaxis views according to the 16-segment model of the American Society of Echocardiography [10,11]. In this model, we placed a sampling point on each segment to record the strain experienced during 3 cardiac cycles. The mean value of each measurement was calculated for further analysis.

The Global Longitudinal Strain (GLS), Circumferential Strain (GCS), and Radial Strain (GRS) were obtained by averaging all the segment strain values.

\section{NT-proBNP Test}

Fasting blood samples were collected from each patient within 24 hours after enrollment. Blood sampling was standardized without tourniquet and immediately centrifuged twice. NT-proBNP was analyzed on a Modular E 170 (Roche Diagnostics, Mannheim, Germany).

\section{Data and statistical analysis}

All quantitative data are expressed as the mean \pm standard deviation (SD) and were analyzed using the SPSS 17.0 statistical software package (IBM Corp., Chicago, IL, USA). Significant differences among groups were analyzed by one-way ANOVA, and pair wise comparisons assessed using two-sample t-tests. Bonferroni-corrected $\mathrm{p}$ values of less than 0.017 were considered statistically significant. The plasma concentration of NT-proBNP was logarithmically converted to log 
NT-proBNP. After transformation, Shapiro Wilk test verified that it conforms to the normal distribution, and was analyzed by analysis of variance. The relationships between VVI parameters and NT- proBNP levels and between VVI parameters and IVSD were assessed by the Pearman correlation coefficient.

Interobserver variability, expressed as a coefficient of variation, was assessed by analyzing $40 \mathrm{VVI}$ movie clips chosen randomly by 2 independent investigators who were blinded to each other's results. For intraobserver variability, 40 VVI movie clips were analyzed twice within an interval of 1 month by one investigator who was blinded to the previous results.

\section{Results}

\section{Clinical characteristics}

There were no significant differences in age, sex, BSA, heart rate, or blood pressure among the 3 groups (Table 1).

\section{Conventional echo parameters}

The IVSD, LVMWT, LAD, LAVI, and LVOT-PG of the HCM patients were significantly higher than those of the patients in the $\mathrm{G}+/ \mathrm{P}$ - group and the control subjects $(\mathrm{P}<0.01)$. Meanwhile, $\mathrm{G}+\mathrm{P}+$ patients had a significantly lower $\mathrm{E} / \mathrm{A}(\mathrm{P}<0.01)$. However, none of the conventional echo parameters were significantly different between the G+/P- group and the control group. In addition, there were no significant differences in LVEDV, LVESV or EF among the three groups (Table 1, Figure 1).

\section{VVI parameters}

Regional longitudinal peak systolic strain: The longitudinal peak systolic strain of the basal inferoseptum and basal anteroseptum in the $\mathrm{G}+/ \mathrm{P}$ - group was significantly lower than that in the control group. The longitudinal peak systolic strain of the basal and middle segments of the inferolatera in the G+/P- group was significantly higher than that in the control group. The peak longitudinal strain of each segment in the $\mathrm{G}+/ \mathrm{P}+$ group was significantly lower than that in the control group and G+/P- group (Table 2, Figures 2 and 3).

Regional circumferential peak systolic strain: There were no significant differences among the normal control, G+/P- and G+/P+ groups for GCS values at all levels (Table 3, Figures 2 and 3).
Table 1: Clinical characteristics and conventional echocardiographic ultrasound parameters for all groups.

\begin{tabular}{|c|c|c|c|c|}
\hline & $\begin{array}{l}\text { Control } \\
(n=48)\end{array}$ & $\begin{array}{c}G+/ P \\
(n=40)\end{array}$ & $\begin{array}{l}G+/ P+ \\
(n=45)\end{array}$ & $\begin{array}{l}\text { P-value for } \\
\text { ANOVA }\end{array}$ \\
\hline Age, y & $39 \pm 17$ & $42 \pm 16$ & $48 \pm 15$ & 0.58 \\
\hline Sex (male/female) & $26 / 22$ & $21 / 19$ & $25 / 20$ & 0.46 \\
\hline BSA, M2 & $1.7 \pm 0.2$ & $1.6 \pm 0.2$ & $1.7 \pm 0.2$ & 0.29 \\
\hline Heart rate, bpm & $71 \pm 18$ & $73 \pm 14$ & $72 \pm 16$ & 0.55 \\
\hline SBP, mmHg & $107 \pm 13$ & $109 \pm 11$ & $108 \pm 12$ & 0.12 \\
\hline DBP, mmHg & $74 \pm 10$ & $73 \pm 11$ & $75 \pm 9$ & 0.19 \\
\hline Mutant gene, \% & & & & 0.52 \\
\hline MYH7 & 0 & $13(44 \%)$ & $9(26 \%)$ & - \\
\hline MYBPC3 & 0 & 15 (50\%) & $22(63 \%)$ & - \\
\hline TNNT2 & 0 & $1(3 \%)$ & $3(9 \%)$ & - \\
\hline TNNI3 & 0 & $1(3 \%)$ & $1(2 \%)$ & - \\
\hline IVSD (mm) & $9.0 \pm 0.9$ & $8.8 \pm 1.0$ & $19.4 \pm 5.2 * \#$ & $<0.01$ \\
\hline LVMWT (mm) & $8.9 \pm 1.0$ & $9.2 \pm 1.4$ & $17.6 \pm 6.8^{*} \#$ & $<0.01$ \\
\hline LAD (mm) & $33 \pm 4$ & $34 \pm 3$ & $41 \pm 5 * \#$ & $<0.01$ \\
\hline LAVI $\left(\mathrm{ml} / \mathrm{m}^{2}\right)$ & $21 \pm 5$ & $22 \pm 4$ & $31 \pm 6 * \#$ & $<0.01$ \\
\hline LVEDV (ml) & $72 \pm 15$ & $73 \pm 16$ & $75 \pm 17$ & 0.09 \\
\hline LVESV (ml) & $28 \pm 6$ & $29 \pm 8$ & $31 \pm 7$ & 0.11 \\
\hline LVOT-PG (mmHg) & $2.2 \pm 0.9$ & $2.5 \pm 0.7$ & $22 \pm 30.5^{*} \#$ & $<0.01$ \\
\hline EF (\%) & $63 \pm 4$ & $63 \pm 5$ & $64 \pm 6$ & 0.21 \\
\hline$E / A$ & $1.4 \pm 0.6$ & $1.4 \pm 0.5$ & $1.0 \pm 0.5^{*} \#$ & $<0.01$ \\
\hline
\end{tabular}

BSA: Body Surface Area; DBP: Diastolic Blood Pressure; SBP: Systolic Blood Pressure; MYH7: Myosin Heavy Chain 7; MYBPC3: Myosin-Binding Protein C3; TNNT2: Cardiac Troponin T; TNNI3: Cardiac Troponin I; IVSD: Interventricular Septum Thickness in Diastolic; LVMWT: Left Ventricular Wall Maximum Thickness; LAD: Left Atrial Anteroposterior Diameter; LAVI: Left Atrial Volume Index; LVEDV: Left Ventricular End-Diastolic Volume; LVESV: Left Ventricular End-Systolic Volume; LVOT-PG: Left Ventricular Outflow Tract Pressure Gradient; EF: Ejection Fraction; E/A: mitral early diastolic filling ratio.

*Significant difference vs. Controls;

\#Significant difference between G+/P-group and G+/P+ group.
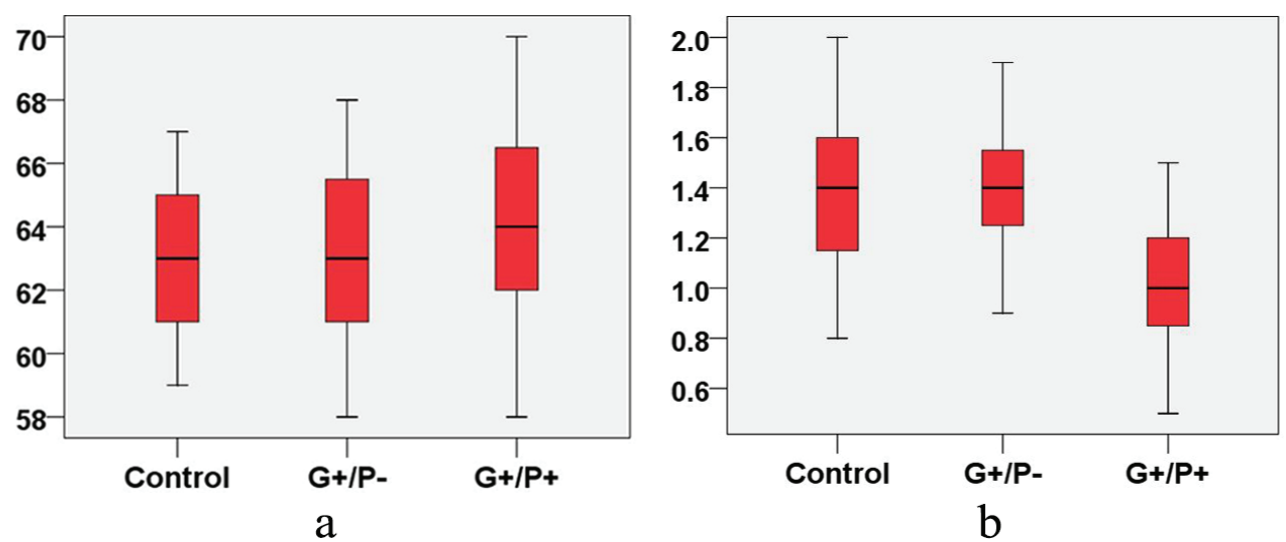

Figure 1: Comparison of conventional echocardiographic ultrasound parameters in the three groups. a. EF; b. E/A. 

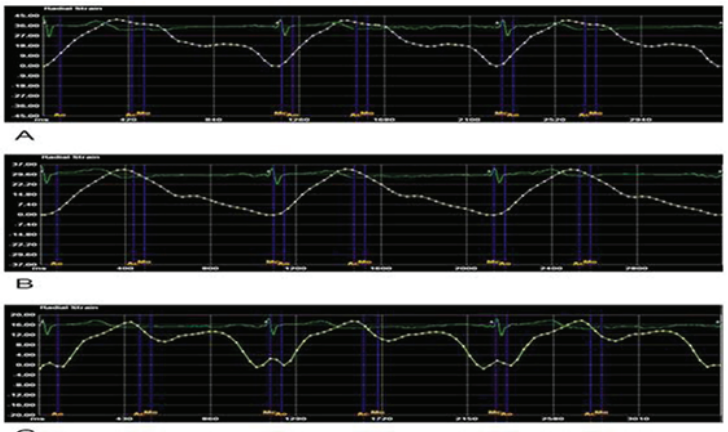

a
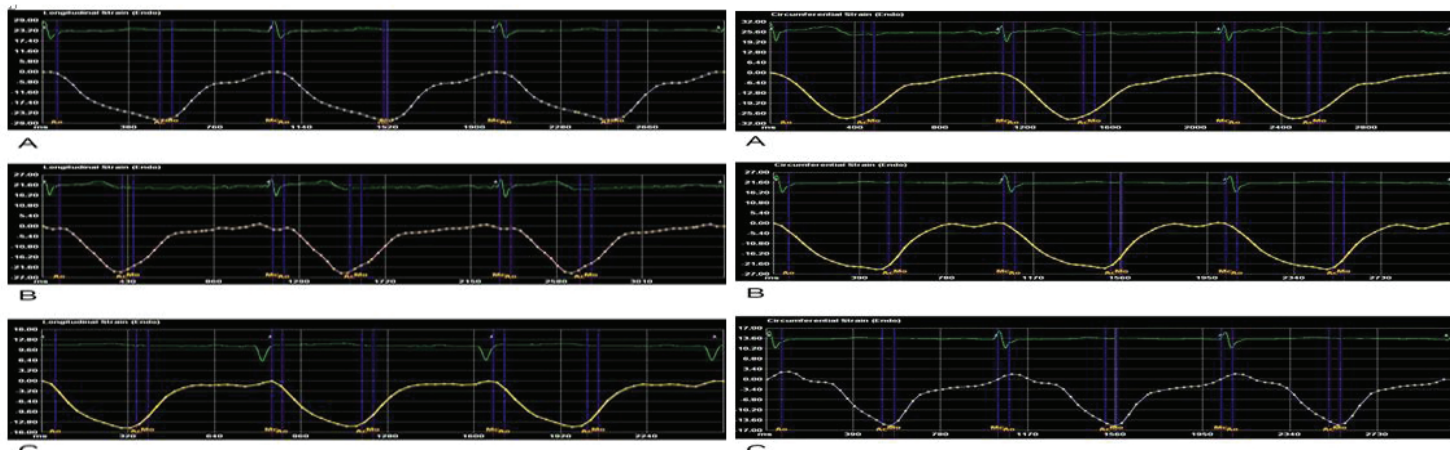

b
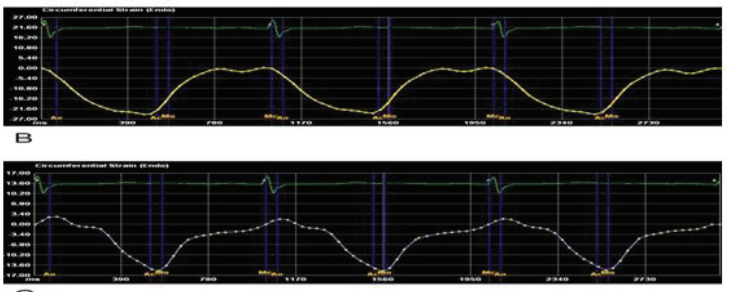

C

Figure 2: Longitudinal, circumferential and radial strain curve for the left ventricle in the three groups. a. radial strain curve; b. Longitudinal strain curve; c. circumferential strain curve. A. Normal control; B. G+/P-; C. G+/P+.

Table 2: Comparison of left ventricular longitudinal peak systolic strain among all groups.

\begin{tabular}{|c|c|c|c|c|}
\hline Left ventricular wall segmentation & Control & G+/P- & $\mathrm{G}+/ \mathrm{P}+$ & P-value \\
\hline \multicolumn{5}{|l|}{ Apical 4-chamber } \\
\hline Basal anterolateral & $-20.31 \pm 7.15$ & $-20.41 \pm 5.61$ & $-13.74 \pm 2.82 * \#$ & $<0.01$ \\
\hline Mid anterolateral & $-20.23 \pm 6.74$ & $-20.55 \pm 6.13$ & $-12.06 \pm 2.94^{*} \#$ & $<0.01$ \\
\hline Apical lateral & $-19.35 \pm 6.94$ & $-19.87 \pm 5.35$ & $-11.84 \pm 2.60 * \#$ & $<0.01$ \\
\hline Basal inferoseptum & $-21.37 \pm 7.55$ & $-15.48 \pm 3.04 *$ & $-8.12 \pm 1.34 * \#$ & $<0.01$ \\
\hline Mid inferoseptum & $-19.86 \pm 7.32$ & $-18.65 \pm 2.72$ & $-8.64 \pm 1.55^{*} \#$ & $<0.01$ \\
\hline Apical septum & $-20.14 \pm 6.76$ & $-20.88 \pm 3.60$ & $-11.86 \pm 1.24 * \#$ & $<0.01$ \\
\hline \multicolumn{5}{|l|}{ Apical 2-chamber } \\
\hline Basal anterior & $-23.35 \pm 7.77$ & $-22.57 \pm 5.64$ & $-10.54 \pm 2.07 * \#$ & $<0.01$ \\
\hline Mid anterior & $-20.26 \pm 5.52$ & $-19.96 \pm 5.35$ & $-9.22 \pm 1.85 * \#$ & $<0.01$ \\
\hline Apical anterior & $-20.74 \pm 6.75$ & $-19.87 \pm 4.91$ & $-11.80 \pm 2.46^{*} \#$ & $<0.01$ \\
\hline Basal inferior & $-20.27 \pm 6.65$ & $-20.12 \pm 5.34$ & $-13.84 \pm 3.17 * \#$ & $<0.01$ \\
\hline Mid inferior & $-19.38 \pm 6.83$ & $-18.68 \pm 6.15$ & $-12.96 \pm 2.95^{*} \#$ & $<0.01$ \\
\hline Apical inferior & $-20.15 \pm 7.31$ & $-19.73 \pm 5.80$ & $-12.15 \pm 3.34 * \#$ & $<0.01$ \\
\hline \multicolumn{5}{|l|}{ Apical 3-chamber } \\
\hline Basal anteroseptum & $-21.23 \pm 7.82$ & $-15.94 \pm 5.54 *$ & $-9.54 \pm 2.04 * \#$ & $<0.01$ \\
\hline Mid anteroseptum & $-20.62 \pm 5.70$ & $-20.65 \pm 4.12$ & $-8.95 \pm 1.86 * \#$ & $<0.01$ \\
\hline Basal inferolateral & $-19.25 \pm 7.85$ & $-22.74 \pm 6.82^{*}$ & $-13.61 \pm 2.74 * \#$ & $<0.01$ \\
\hline Mid inferolateral & $-18.24 \pm 6.40$ & $-21.12 \pm 5.94 *$ & $-12.42 \pm 1.62 * \#$ & $<0.01$ \\
\hline
\end{tabular}

*Significant difference vs. Controls;

\#Significant difference between G+/P-group and G+/P+ group. 
Table 3: Comparison of left ventricular radial peak systolic strain among all groups.

\begin{tabular}{|c|c|c|c|c|}
\hline Left ventricular wall segmentation & Control & G+/P- & $\mathbf{G}+/ \mathbf{P +}$ & P-value \\
\hline \multicolumn{5}{|l|}{ Mitral valve level } \\
\hline Anteroseptum & $-27.81 \pm 6.65$ & $-27.74 \pm 6.25$ & $-26.77 \pm 7.13$ & 0.61 \\
\hline Anterior wall & $-31.4 \pm 8.62$ & $-29.34 \pm 7.63$ & $-29.82 \pm 8.74$ & 0.55 \\
\hline Anterolateral wall & $-31.61 \pm 5.87$ & $-30.97 \pm 7.86$ & $-32.15 \pm 8.33$ & 0.49 \\
\hline Inferolateral wall & $-27.91 \pm 7.46$ & $-26.72 \pm 9.41$ & $-27.33 \pm 4.17$ & 0.81 \\
\hline Inferior wall & $-25.23 \pm 5.17$ & $-26.23 \pm 6.41$ & $-27.41 \pm 5.80$ & 0.68 \\
\hline Inferoseptum Papillary muscle level & $-28.32 \pm 8.77$ & $-26.73 \pm 9.74$ & $-26.83 \pm 11.26$ & 0.59 \\
\hline Anteroseptum & $-26.52 \pm 5.23$ & $-27.41 \pm 5.74$ & $-26.12 \pm 5.13$ & 0.72 \\
\hline Anterior wall & $-32.65 \pm 6.58$ & $-31.52 \pm 6.51$ & $-30.89 \pm 8.63$ & 0.69 \\
\hline Anterolateral wall & $-32.43 \pm 6.76$ & $-32.73 \pm 7.65$ & $-33.36 \pm 6.84$ & 0.53 \\
\hline Inferolateral wall & $-29.30 \pm 9.14$ & $-28.24 \pm 8.25$ & $-29.72 \pm 5.97$ & 0.49 \\
\hline Inferior wall & $-26.75 \pm 5.95$ & $-25.76 \pm 5.86$ & $-27.36 \pm 5.35$ & 0.67 \\
\hline $\begin{array}{l}\text { Inferoseptum } \\
\text { Apical level }\end{array}$ & $-29.13 \pm 7.63$ & $-30.86 \pm 6.36$ & $-29.62 \pm 6.50$ & 0.58 \\
\hline Anteroseptum & $-40.74 \pm 5.97$ & $-40.33 \pm 5.52$ & $-38.23 \pm 9.41$ & 0.64 \\
\hline Anterior wall & $-41.85 \pm 7.94$ & $-40.64 \pm 6.84$ & $-39.12 \pm 10.24$ & 0.79 \\
\hline Anterolateral wall & $-42.65 \pm 7.24$ & $-41.40 \pm 6.94$ & $-40.80 \pm 7.74$ & 0.58 \\
\hline Inferior wall & $-41.87 \pm 6.25$ & $-40.29 \pm 6.04$ & $-39.82 \pm 8.51$ & 0.62 \\
\hline
\end{tabular}

*Significant difference vs. Controls;

\#Significant difference between $\mathrm{G}+/ \mathrm{P}$ - group and $\mathrm{G}+/ \mathrm{P}+$ group.

Table 4: Comparison of left ventricular radial peak systolic strain among all groups.

\begin{tabular}{|c|c|c|c|c|}
\hline Left ventricular wall segmentation & Control & G+/P- & $\mathrm{G}+/ \mathrm{P}+$ & P-value \\
\hline \multicolumn{5}{|l|}{ Mitral valve level } \\
\hline Anteroseptum & $37.91 \pm 4.72$ & $36.76 \pm 4.76$ & $26.86 \pm 7.93^{*} \#$ & $<0.01$ \\
\hline Anterior wall & $38.55 \pm 5.43$ & $37.45 \pm 5.37$ & $25.44 \pm 10.45 * \#$ & $<0.01$ \\
\hline Anterolateral wall & $37.95 \pm 7.14$ & $37.19 \pm 7.42$ & $32.72 \pm 7.67 * \#$ & $<0.05$ \\
\hline Inferolateral wall & $36.96 \pm 6.52$ & $36.34 \pm 6.04$ & $30.76 \pm 7.15^{*} \#$ & $<0.05$ \\
\hline Inferior wall & $37.93 \pm 9.31$ & $37.67 \pm 9.16$ & $31.74 \pm 10.04 * \#$ & $<0.05$ \\
\hline Inferoseptum Papillary muscle level & $36.46 \pm 8.17$ & $35.37 \pm 8.27$ & $25.22 \pm 11.42 * \#$ & $<0.01$ \\
\hline Anteroseptum & $43.64 \pm 8.15$ & $41.49 \pm 8.49$ & $33.17 \pm 10.45 * \#$ & $<0.01$ \\
\hline Anterior wall & $43.33 \pm 7.33$ & $42.97 \pm 5.37$ & $33.46 \pm 9.93 * \#$ & $<0.01$ \\
\hline Anterolateral wall & $43.77 \pm 7.32$ & $43.24 \pm 7.34$ & $37.27 \pm 7.15^{*} \#$ & $<0.01$ \\
\hline Inferolateral wall & $41.24 \pm 8.20$ & $41.45 \pm 8.37$ & $36.12 \pm 8.52 * \#$ & $<0.01$ \\
\hline Inferior wall & $43.11 \pm 8.42$ & $42.14 \pm 8.37$ & $37.53 \pm 7.31 * \#$ & $<0.01$ \\
\hline $\begin{array}{l}\text { Inferoseptum } \\
\text { Apical level }\end{array}$ & $42.42 \pm 8.52$ & $42.79 \pm 8.46$ & $31.16 \pm 8.23^{*} \#$ & $<0.01$ \\
\hline Anteroseptum & $36.56 \pm 4.89$ & $37.26 \pm 8.37$ & $26.12 \pm 11.26 * \#$ & $<0.01$ \\
\hline Anterior wall & $35.41 \pm 5.32$ & $36.43 \pm 7.43$ & $26.62 \pm 10.23 * \#$ & $<0.01$ \\
\hline Anterolateral wall & $34.40 \pm 7.67$ & $35.45 \pm 7.04$ & $28.23 \pm 8.26 * \#$ & $<0.05$ \\
\hline Inferior wall & $35.40 \pm 8.42$ & $36.16 \pm 8.45$ & $27.72 \pm 11.01 * \#$ & $<0.01$ \\
\hline
\end{tabular}

*Significant difference vs. Controls;

\#Significant difference between $\mathrm{G}+/ \mathrm{P}$ - group and $\mathrm{G}+/ \mathrm{P}+$ group.

Regional radial peak systolic strain: In the G+/P- group, the peak radial strain at all levels was not significantly different from that of the control group. The peak radial strain of each segment in the $\mathrm{G}+$ / $\mathrm{P}+$ group was significantly lower than those in the control and G+/Pgroups (Table 4, Figures 2 and 3).

Global longitudinal, circumferential and radial strain: There was no significant difference in systolic longitudinal, circumferential, or radial strain between the G+/P- group and the control group. The systolic GLS of the $\mathrm{G}+/ \mathrm{P}+$ group was lower than that of the control group and the G+/P- group, and the difference was very significant
$(\mathrm{P}<0.01)$. The systolic GRS of the $\mathrm{G}+/ \mathrm{P}+$ group was lower than that of the control group and the G+/P- group, and the difference was significant $(\mathrm{P}<0.05)$. The systolic GCS of the $\mathrm{G}+/ \mathrm{P}+$ group was not significantly different from that of the control group or the G+/Pgroup (Table 5, Figure 3).

NT-proBNP level: NT-proBNP levels were significantly higher in $\mathrm{HCM}$ patients than in the control group and G+/P-group. There were no detectable differences in $\mathrm{G}+/ \mathrm{P}$ - individuals compared with healthy controls (Table 5). 


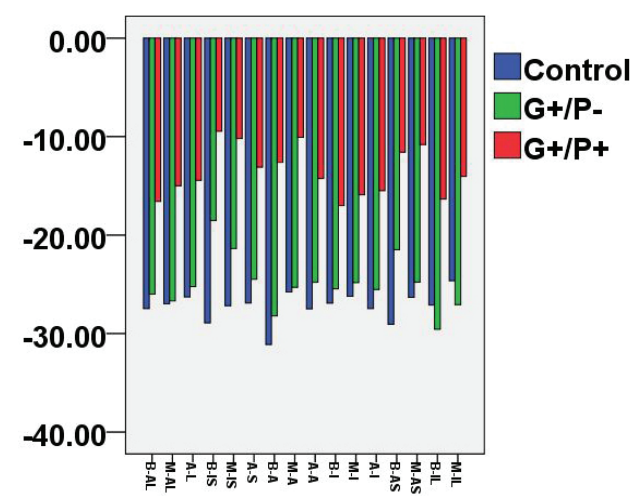

a

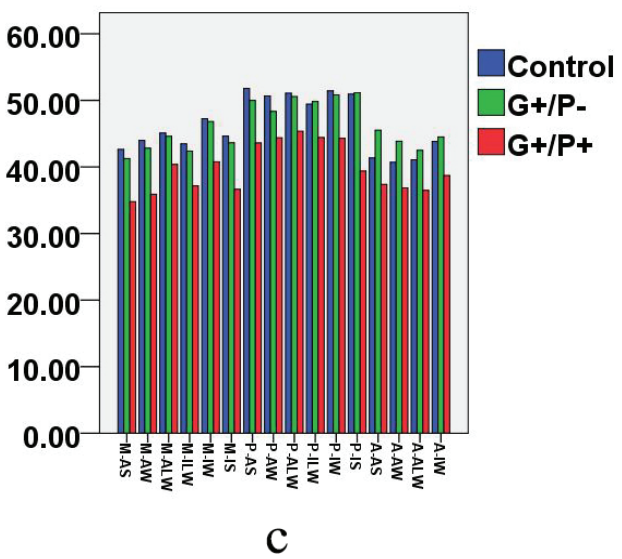

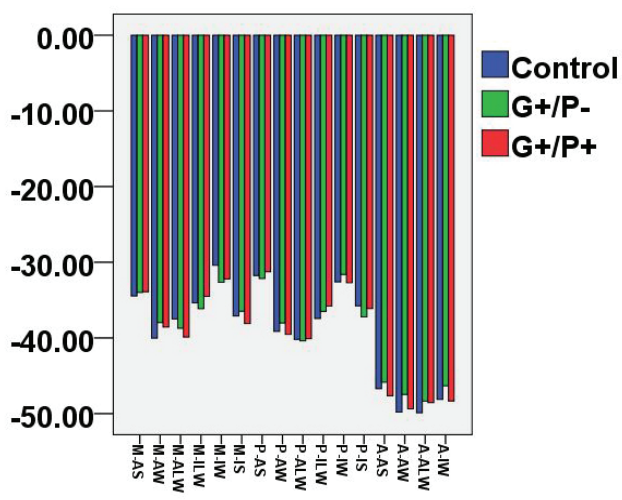

b

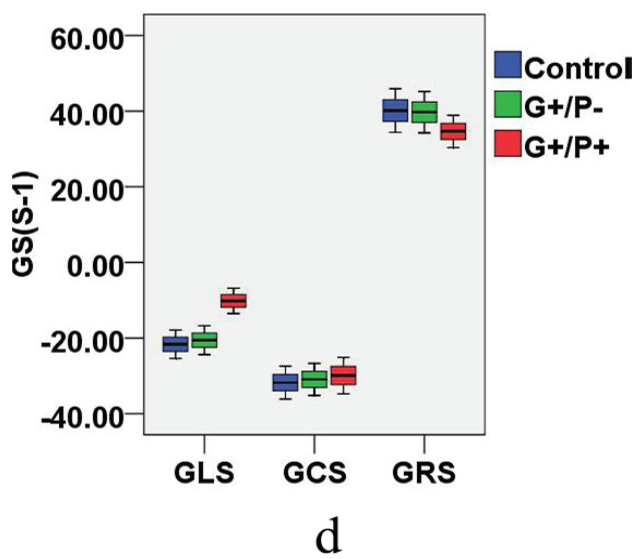

Figure 3: Longitudinal, circumferential and radial strain of 16 segments and global systolic strain in the three groups. a. Longitudinal strain; b. circumferential strain; c. radial strain; d. global strain.

\section{Correlations of analysis results}

In the HCM patients, NT-proBNP was correlated with greater IVSD ( $r=0.545, P=0.004)$, lower GLS ( $r=0.566, P=0.003)$, but no association with GRS. IVSD was correlated with lower GLS ( $\mathrm{r}=0.545, \mathrm{P}=0.003)$, lower GRS ( $\mathrm{r}=0.37, \mathrm{P}=0.031$ ), but no association with GCS (Figure 4).

\section{Repeatability test}

The interobserver correlation coefficients for GLS estimates of 42 - and 3-chamber views were $0.45(\mathrm{P}=0.039), 0.52(\mathrm{P}=0.043)$, and 0.48 $(\mathrm{P}=0.027)$, respectively. The interobserver correlation coefficients for GRS estimates of 4-, 2- and 3-chamber views were $0.43(\mathrm{P}=0.041)$, $0.46(\mathrm{P}=0.048)$, and $0.51(\mathrm{P}=0.029)$, respectively. The interobserver correlation coefficients for GCS estimates at the level of the mitral valve, papillary muscle, and apex were $0.68(\mathrm{P}=0.002), 0.71(\mathrm{P}=0.001)$, and $0.62(\mathrm{P}=0.004)$, respectively.

The intraobserver correlation coefficients for GLS dependency estimates of 4-, 2- and 3-chamber views were $0.35(\mathrm{P}=0.183)$, $0.32(\mathrm{P}=0.126)$, and $0.21 \quad(\mathrm{P}=0.38)$, respectively. The intraobserver correlation coefficients for GRS dependency estimates of 4-, 2- and 3 -chamber views were $0.33(\mathrm{P}=0.187), 0.36(\mathrm{P}=0.133)$, and 0.19 $(\mathrm{P}=0.43)$, respectively. The intraobserver correlation coefficients for GCS dependency estimates at the level of the mitral valve, papillary muscle, and apex were $0.71(\mathrm{P}=0.003), 0.59(\mathrm{P}=0.019)$, and 0.64 $(\mathrm{P}=0.047)$, respectively.

\section{Discussion}

In recent years, studies have shown that the primary cause of familial $\mathrm{HCM}$ is mutations in the genes encoding sarcomere proteins and other modification genes. Most of the mutations are in genes encoding sarcomere proteins, and point mutations of the $\beta$-Myosin Heavy Chain gene (MYH7), Myosin Binding Protein C (MYBPC3), Troponin T (TNNT2) and Troponin I (TNNI3) are relatively common [12-16]. Abnormal genetic regulation can lead to the disordered arrangement of myocardial cells and abnormal thickening of the myocardium [1719] and can change calcium sensitivity and muscle fiber tension, thus affecting myocardial contractile and diastolic function.

At present, gene detection is the best method to judge the risk of HCM first-degree relatives, but the premise is to identify the mutated genes that cause HCM carried by the probands in the family, and the screening cycle is long and expensive. Only $50 \% \sim 60 \%$ can be identified. There is an urgent clinical need to identify early indicators that can independently diagnose HCM and assess its risk before LVH.

In this study, subjects were analyzed from longitudinal, radial and circumferential viewpoints. The results showed that there were no significant differences in the global longitudinal, circumferential or radial strains of the systolic period of the LV between the mutation gene carriers and the control group, while the longitudinal strain of the basal inferoseptum and basal anteroseptum was significantly lower, and the longitudinal strain of the basal and mid inferolateral 

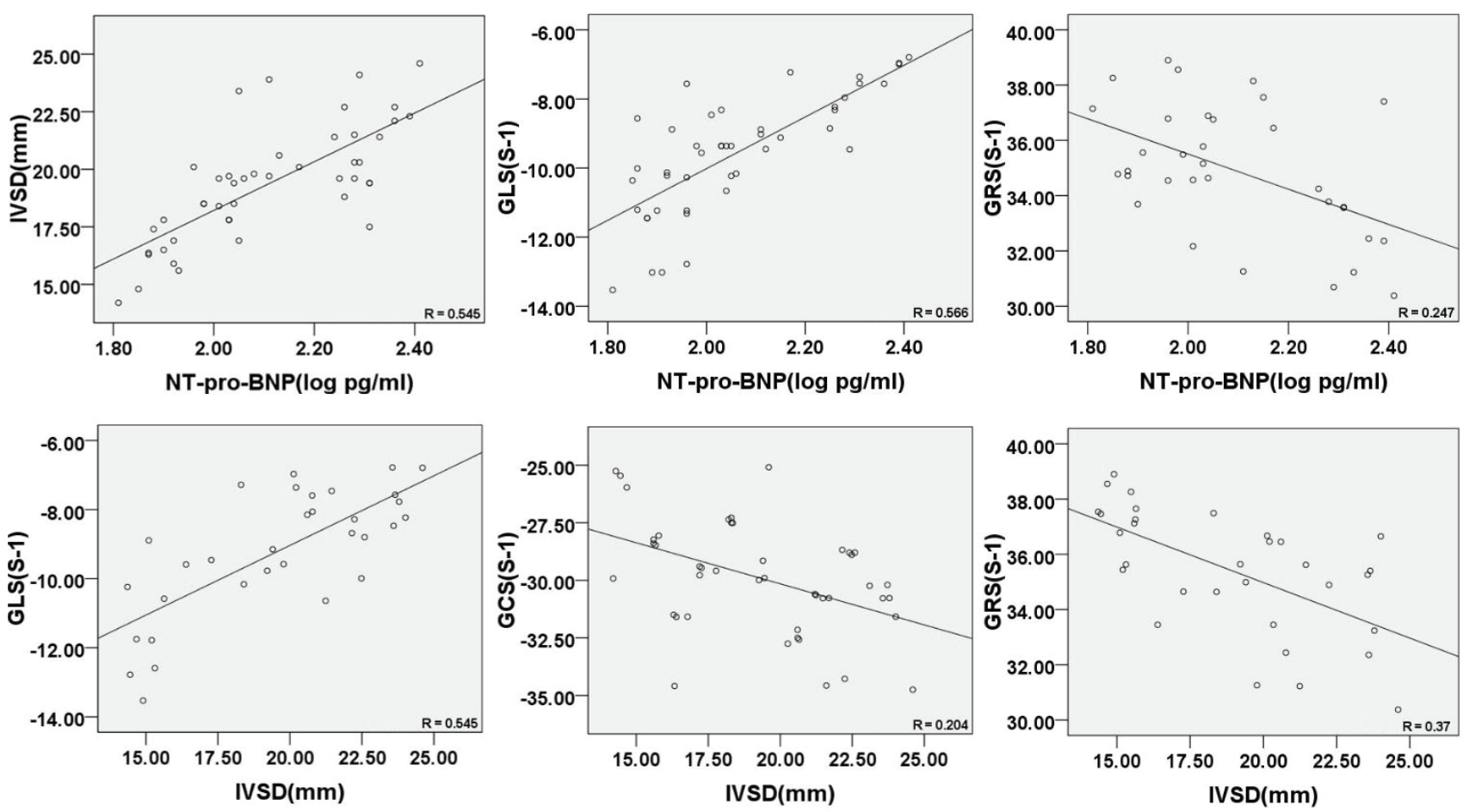

Figure 4: Correlation of N-terminal pro-brain natriuretic peptide (NT-proBNP) levels and IVSD with LV remodeling parameters in the HCM group.

Table 5: Comparison of left ventricular global systemic strain and NT proBNP level among all groups.

\begin{tabular}{|l|c|c|c|c|}
\hline \multicolumn{1}{|c|}{ VVI parameters } & Control & G+/P- & G+/P+ & P-value \\
\hline GLS & $-21.64 \pm 3.76$ & $-20.56 \pm 3.82$ & $-10.16 \pm 3.37 * \#$ & $<0.01$ \\
\hline GCS & $-31.78 \pm 4.37$ & $-30.94 \pm 4.24$ & $-29.92 \pm 4.83$ & 0.32 \\
\hline GRS & $40.16 \pm 5.75$ & $39.73 \pm 5.46$ & $34.64 \pm 4.26 * \#$ & $<0.05$ \\
\hline NT-proBNP (log pg/mL) & $1.51 \pm 0.28$ & $1.64 \pm 0.27$ & $2.06 \pm 0.35 * \#$ & $<0.05$ \\
\hline
\end{tabular}

GLS: Global Longitudinal Strain; GCS: Global Circumferential Strain; GRS: Global Radial Strain; NT-proBNP: N-Terminal pro-Brain Natriuretic Peptide. *Significant difference vs. Controls;

\#Significant difference between $\mathrm{G}+/ \mathrm{P}$ - group and G+/P+ group.

was significantly higher than those of the normal control group. This indicates that regional myocardial segmental systolic function was impaired in carriers of HCM sarcomere gene mutations, and the impairment was limited to the inferoseptum and anteroseptum basal segment. The elevation of longitudinal strain of the inferolateral remains unclear. Perhaps regional myocardium experiencing higher longitudinal strain occurs as a cause of adjacent myocardial deformity (with lower strain). Germans T, et al., [20] found that in HCM gene mutation carriers who did not have ventricular wall hypertrophy, even if the results of conventional echocardiography and ECG were normal, cardiac magnetic resonance technology detected that $81 \%$ of HCM gene mutation carriers had a recess in the basal and intermediate segments of the interventricular septum, which may indicate early disease in HCM gene mutation carriers that will eventually develop HCM. Germans T, et al. also found that the abnormal myocardial structure of carriers of the HCM gene involved local myocardial segments rather than all myocardial segments, and the interventricular septum was the most obviously involved.
HCM gene mutation carriers exhibit disordered arrangement and degeneration of cardiac myocytes, mild fibrosis in the intercellular matrix and increased myocardial stiffness, and the longitudinal myocardial fibers under the endocardium of these patients are more prone to interstitial fibrosis [21]. The regional radial systolic strain (the basal inferoseptum and basal anteroseptum) of the G+/P- group remained similar to that of the control group. This may be because changes in LV radial systolic function occur later than changes in longitudinal systolic function.

Our study showed that NT-proBNP levels in HCM patients were significantly higher and correlated with myocardial deformation and interventricular septal thickness. Among genotype-negative individuals, we also found that there were no differences in NTproBNP concentrations compared with control relatives, but their local segmental deformation parameters were different, which was different from Silva D, et al., [22] who identified mutation carriers of hypertrophic cardiomyopathy by tissue Doppler imaging. 


\section{Conclusions}

In conclusion, the GLS and GRS were diminished in HCM subjects, whereas a compensatory mechanism existed that tended to maintain the GCS. Although the GLS, GRS and GCS of HCM gene mutation carriers were still within the normal range, the longitudinal strain of local myocardial segments was diminished.

VVI can provide quantitative information for the early diagnosis of HCM sarcomere gene mutation carriers without myocardial hypertrophy to improve early diagnosis and identification, and primary prevention and reduction of unnecessary treatment and longterm complications can not only improve the health level of the whole population, but also make rational use of medical resources.

\section{Limitations}

Our study is limited by the small sample size, and larger study populations would be required to further delineate the sensitivity and specificity of this technique. Prospective follow-up is necessary to evaluate the clinical significance of longitudinal strain in $\mathrm{G}+/ \mathrm{P}$ patients.

\section{Ethical Statement}

All protocols pertaining to human subjects were first approved by the Institutional Ethics Committee of Second Xiangya Hospital of Central South University.

\section{Acknowledgments}

We wish to thank all the patients and control subjects for their participation in this study.

\section{Conflict of Interest}

$\mathrm{Li} \mathrm{Yu} \mathrm{declares} \mathrm{that} \mathrm{she} \mathrm{has} \mathrm{no} \mathrm{conflicts} \mathrm{of} \mathrm{interest.} \mathrm{Qichang} \mathrm{Zhou}$ declares that he has no conflicts of interest. Xiangdang Long declares that she has no conflicts of interest. Qinghai Peng declares that he has no conflicts of interest. Zurong Yang declares that she has no conflicts of interest.

\section{References}

1. Authors/Task Force members, Elliott PM, Anastasakis A, Borger MA, Borggrefe M, et al. (2014) 2014 ESC Guidelines on diagnosis and management of hypertrophic cardiomyopathy: the Task Force for the Diagnosis and Management of Hypertrophic Cardiomyopathy of the European Society of Cardiology (ESC). Eur Heart J 35: 2733-2779.

2. Lorenzini M, Norrish G, Field E, Ochoa JP, Cicerchia M, et al. (2020) Penetrance of Hypertrophic Cardiomyopathy in Sarcomere Protein Mutation Carriers. J Am Coll Cardiol 76: 550-559.

3. Bansal M, Cho GY, Chan J, Leano R, Haluska BA, et al. (2008) Feasibility and accuracy of different techniques of two-dimensional speckle based strain and validation with harmonic phase magnetic resonance imaging. J Am Soc Echocardiogr 21: 1318-1325.

4. Wei Z, Su H, Zhang H, Zhou X, Zhu T, et al. (2008) Assessment of left ventricular wall motion in diabetic rats using velocity vector imaging combined with stress echocardiography. Echocardiography 25: 609616.

5. Bohs LN, Geiman BJ, Anderson ME, Gebhart SC, Trahey GE (2000) Speckle tracking for multi-dimensional flow estimation. Ultrasonics 38: 369-375.
6. Langeland S, D'hooge J, Wouters PF, Leather HA, Claus $P$, et al. (2005) Experimental validation of a new ultrasound method for the simultaneous assessment of radial and longitudinal myocardial deformation independent of insonation angle. Circulation 112: 2157-2162.

7. Pirat B, McCulloch ML, Zoghbi WA (2006) Evaluation of global and regional right ventricular systolic function in patients with pulmonary hypertension using a novel speckle tracking method. Am J Cardiol 98: 699-704.

8. Zethelius B, Berglund L, Sundström J, Ingelsson E, Basu S, et al. (2008) Use of multiple biomarkers to improve the prediction of death from cardiovascular causes. N Engl J Med 358: 2107-2116.

9. Lang RM, Badano LP, Mor-Avi V, Afilalo J, Armstrong A, et al. (2015) Recommendations for cardiac chamber quantification by echocardiography in adults: an update from the American Society of Echocardiography and the European Association of Cardiovascular Imaging. J Am Soc Echocardiogr 28: 1-39.

10. Lang RM, Bierig M, Devereux RB, Flachskampf FA, Foster E, et al. (2005) Recommendations for chamber quantification: a report from the American Society of Echocardiography's Guidelines and Standards Committee and the Chamber Quantification Writing Group, developed in conjunction with the European Association of Echocardiography, a branch of the European Society of Cardiology. J Am Soc Echocardiogr 18: 1440-1463.

11. Schiller NB, Shah PM, Crawford M, DeMaria A, Devereux R, et al. (1989) Recommendations for quantitation of the left ventricle by two- dimensional echocardiography. American Society of Echocardiography Committee on standards, subcommittee on quantitation of two-dimensional echocardiograms. J Am Soc Echocardiogr 2: 358-367.

12. Girolami F, Olivotto I, Passerini I, Zachara E, Nistri S, et al. (2006) A molecular screening strategy based on $\beta$-myosin heavy chain, cardiac myosin binding protein $C$ and troponin $T$ genes in Italian patients with hypertrophic cardiomyopathy. J Cardio Med (Hagerstown) 7: 601-607.

13. Niimura H, Patton KK, McKenna WJ, Soults J, Maron BJ, et al. (2002) Sarcomere protein gene mutations in hypertrophic cardiomyopathy of the elderly. Circulation 105: 446-451.

14. Watkins $H$, Conner D, Thierfelder L, Jarcho JA, MacRae $C$, et al. (1995) Mutations in the cardiac myosin binding protein-Cgene on chromosome 11 cause familial hypertrophic cardiomyopathy. Nat Genet 11: 434-437.

15. Fananapazir L, Epstein ND (1995) Prevalence of hypertrophic cardiomyopathy and limitations of screening methods. Circulation 92: 700-704.

16. Maron BJ, Gardin JM, Flack JM, Gidding SS, Kurosaki TT, et al. (1995) Prevalence of hypertrophic cardiomyopathy in a general population of young adults. Echocardiographic analysis of 4111 subjects in the CARDIA Study. Coronary Artery Risk Development in (Young) Adults. Circulation 92: 785-789.

17. Hulot JS, Fauconnier J, Ramanujam D, Chaanine A, Aubart F, et al. (2011) Critical role for stromal interaction molecule1in cardiac hypertrophy. Circulation 124: 796-805.

18. Kho C, Lee A, Jeong D, Hajjar RJ (2010) Refilling Intracellular Calcium Stores. Drug Discov Today Dis Mech 7: e145-e150.

19. Kojima A, Kitagawa $H$, Omatsu-Kanbe M, Matsuura $H$, Nosaka $S$ (2010) Ca2+ paradox injury mediated through TRPC channels in mouse ventricular myocytes. Br J Pharmacol 161: 1734-1750. 
20. Germans T, Wilde AA, Dijkmans PA, Chai W, Kamp O, et al. (2006) Structural abnormalities of the inferoseptal left ventricular wall detected by cardiac magnetic resonance imaging in carriers of hypertrophic cardiomyopathy mutations. J Am Coll Cardiol 48: 25182523.

21. Spindler M, Saupe KW, Christe ME, Sweeney HL, Seidman $C E$, et al. (1998) Diastolic dysfunction and altered energetics in the alphaMHC403/+ mouse model of familial hypertrophic cardiomyopathy. J Clin Invest 101: 1775-1783.

22. Silva D, Madeira H, Almeida A, Brito D (2013) Tissue Doppler imaging and plasma $\mathrm{N}$-terminal probrain natriuretic peptide for the identification of hypertrophic cardiomyopathy mutation carriers. Am J Cardiol 112: 996-1004. 